\title{
Clinical Trial Startup Material
}

National Cancer Institute

\section{Source}

National Cancer Institute. Clinical Trial Startup Material. NCI Thesaurus. Code 6115605.

A collection of agendas, presentation materials, and other documents related to a clinical trial startup. 